01

\title{
Двухкомпонентный векторный бризер
}

\author{
(ㄷ Г.Т. Адамашвили \\ Грузинский технический университет, Тбилиси, Грузия \\ E-mail: guram_adamashvili@ymail.com \\ Поступило в Редакцию 14 августа 2020г. \\ В окончательной редакции 14 августа 2020г. \\ Принято к публикации 3 марта 2021 г.
}

Рассматривается двухкомпонентное решение модифицированного уравнения Бенджамина-Бона-Махони. С помощью обобщенной версии пертурбативного метода редукции уравнение трансформируется к связанным нелинейным уравнениям Шредингера для вспомогательных функций. Получено явное аналитическое выражение для формы и параметров двухкомпонентного векторного бризера, компоненты которого осциллируют на суммарной и разностной частотах и волновых числах.

Ключевые слова: двухкомпонентные векторные бризеры, модифицированное уравнение ВВМ, обобщенный пертурбативный метод редукции.

DOI: 10.21883/PJTF.2021.11.51000.18511

Нелинейные уединенные волны, такие как бризеры и их различные разновидности, являются одним из основных объектов исследования в теории нелинейных волн. Хотя эти волны встречаются в совершенно разных материалах и описывают различные физические явления, многие их общие свойства идентичны. Нелинейные уединенные волны можно разделить на два основных типа: однокомпонентные и двухкомпонентные уединенные волны. Свойства и методы исследования каждого из них различны: методы изучения однокомпонентных волн совершенно неприемлемы для изучения двухкомпонентных уединенных волн. Это связано с тем, что для изучения двухкомпонентных волн требуется большее количество вспомогательных функций и параметров. Ввиду того что число нелинейных уравнений, полностью интегрируемых методом обратной задачи [1], как для однокомпонентных, так и для двухкомпонентных уединенных волн весьма ограничено, используют различные асимптотические методы. В частности, пертурбативный метод редукции (ПМР) адаптирован для изучения однокомпонентных уединенных волн, в этом случае используют одну комплексную вспомогательную функцию и два постоянных параметра [2]. В отличие от ПМР в его обобщенной версии используются две комплексные вспомогательные функции и восемь постоянных параметров, что позволяет исследовать двухкомпонентные нелинейные уединенные волны $[3,4]$.

Волновые уравнения в нелинейной оптике и нелинейной акустике для медленных огибающих функции содержат их первые и вторые производные по пространственным координатам и времени. Первые производные члены описывают формирование однокомпонентных солитонов и бризеров, в то время как относительно малые вторые производные члены (по сравнению с первыми производными) могут описать образование связанных состояний двух однокомпонентных бризеров, движущихся как единое целое, и обеспечивают формирование двухкомпонентного векторного бризера. При этом одна компонента такого векторного импульса осциллирует на суммарной, а вторая - на разностной частоте и волновых числах. Именно такая ситуация реализуется для оптических и акустических резонансных двухкомпонентных векторных бризеров самоиндуцированной прозрачности - векторных 0л-импульсов $[3,4]$. Изучение нерезонансных нелинейных волн в диспергирующей и нелинейной среде керровского типа привело к аналогичным результатам: формированию двухкомпонентного векторного бризера, осциллирующего на суммарной и разностной частотах и волновых числах (СРЧ) [5].

Учитывая математическую аналогию между акустическими и электромагнитными волнами, интересно рассмотреть совершенно другие явления и соответствующие им уравнения, чем нелинейные уравнения, изучаемые в нелинейной оптике и нелинейной акустике, а именно рассмотреть поверхностные волны в диспергирующих нелинейных средах, ангармонические фононы в кристаллах, акустогравитационные волны в тонких слоях жидкости, нелинейные явления в плазме, нелинейные волны в определенном классе акустических метаматериалов „масса-в-массе“ и т. Д. Для описания этих явлений используют уравнение Бенджамина-Бона-Махони (BВМ) и его модифицированную версию.

Однокомпонентные нелинейные волны модифицированного уравнения ВВМ широко исследованы [6-10]. Однако свойства и методы исследования двухкомпонентного векторного бризера, осциллирующего на СРЧ модифицированного уравнения ВВМ, качественно отличаются от свойств и методов исследования однокомпонентных солитона и бризера этого уравнения. Цель настоящей работы - доказать, что модифицированное уравнение ВВМ

$$
u_{t}+C u_{z}+\beta u_{z z t}+a u^{2} u_{z}=0
$$

имеет решение в виде двухкомпонентного векторного бризера, осциллирующего на СРЧ, который не был 
исследован до сих пор. Здесь $u(z, t)$ - действительная функция, $C, \beta$ и $a-$ произвольные постоянные.

Уравнение (1) можно упростить, используя метод медленно меняющейся огибающей. Для этого функцию $u(z, t)$ можно представить в виде

$$
u=\sum_{l= \pm 1} \hat{u}_{l} e^{i l(k z-\omega t)}
$$

где $\hat{u}_{l}(z, t)$ - медленно меняющаяся комплексная амплитуда. Подставляя уравнение (2) в нелинейное уравнение (1), получаем закон дисперсии импульса в среде

$$
\omega=\frac{C k}{1-\beta k^{2}}
$$

Для исследования двухкомпонентного решения уравнения (1) мы используем обобщенный ПМР [3-5], с помощью которого функцию $\hat{u}_{l}$ можно представить как

$$
\hat{u}_{l}(z, t)=\sum_{\alpha=1}^{\infty} \sum_{n=-\infty}^{\infty} \varepsilon^{\alpha} Y_{l, n} f_{l, n}^{(\alpha)}\left(\xi_{l, n}, \tau\right),
$$

где

$$
\begin{gathered}
Y_{l, n}=e^{i n\left(Q_{l, n} z-\Omega_{l, n} t\right)}, \quad \xi_{l, n}=\varepsilon Q_{l, n}\left(z-v_{l, n} t\right), \\
\tau=\varepsilon^{2} t, \quad v_{l, n}=\frac{d \Omega_{l, n}}{d Q_{l, n}},
\end{gathered}
$$

$\varepsilon$ - малый параметр. Такое разложение позволяет выделить из $\hat{u}_{l}$ еще более медленно меняющиеся вспомогательные функции $f_{l, n}^{(\alpha)}$. Предполагается, что величины $\Omega_{l, n}, Q_{l, n}$ и $f_{l, n}^{(\alpha)}$ удовлетворяют неравенствам $\omega \gg \Omega_{l, n}$, $k \gg Q_{l, n}$,

$$
\begin{aligned}
&\left|\frac{\partial f_{l, n}^{(\alpha)}}{\partial t}\right| \ll \Omega_{l, n}\left|f_{l, n}^{(\alpha)}\right|, \\
&\left|\frac{\partial f_{l, n}^{(\alpha)}}{\partial z}\right| \ll Q_{l, n}\left|f_{l, n}^{(\alpha)}\right|
\end{aligned}
$$

для любых значений индексов $l$ и $n$.

Подставляя уравнения (2) и (4) в уравнение (1) и приравнивая к нулю члены с одинаковыми степенями $\varepsilon$, мы можем получить связанные нелинейные уравнения Шредингера для вспомогательных функции $\lambda_{ \pm}=\varepsilon f_{+1, \pm 1}^{(1)}$ в виде

$$
i\left(\frac{\partial \lambda_{ \pm}}{\partial t}+v_{ \pm} \frac{\partial \lambda_{ \pm}}{\partial z}\right)+p_{ \pm} \frac{\partial^{2} \lambda_{ \pm}}{\partial z^{2}}+q_{ \pm}\left(\left|\lambda_{ \pm}\right|^{2}+2\left|\lambda_{\mp}\right|^{2}\right) \lambda_{ \pm}=0,
$$

а также определить связь между величинами $\Omega_{l, n}$ и $Q_{l, n}$. Когда $l=n= \pm 1$, тогда $f_{ \pm 1, \pm 1}^{(1)} \neq 0$, и имеем уравнение

$$
\begin{gathered}
\left(\beta k^{2}-1\right) \Omega_{ \pm 1, \pm 1}+(C+2 \beta k \omega) Q_{ \pm 1, \pm 1}+2 \beta k \Omega_{ \pm 1, \pm 1} Q_{ \pm 1, \pm 1} \\
+\beta \omega Q_{ \pm 1, \pm 1}^{2}+\beta Q_{ \pm 1, \pm 1}^{2} \Omega_{ \pm 1, \pm 1}=0
\end{gathered}
$$

а когда $l=-n= \pm 1$, тогда $f_{ \pm 1, \mp 1}^{(1)} \neq 0$, и мы получаем следующее уравнение:

$$
\begin{gathered}
\left(\beta k^{2}-1\right) \Omega_{ \pm 1, \mp 1}+(C+2 \beta k \omega) Q_{ \pm 1, \mp 1}-2 \beta k \Omega_{ \pm 1, \mp 1} Q_{ \pm 1, \mp 1} \\
-\beta \omega Q_{ \pm 1, \mp 1}^{2}+\beta Q_{ \pm 1, \mp 1}^{2} \Omega_{ \pm 1, \mp 1}=0,
\end{gathered}
$$

где

$$
\begin{gathered}
p_{ \pm}=\beta \frac{2\left(k \pm Q_{ \pm 1}\right) v_{ \pm}+\left(\omega \pm \Omega_{ \pm 1}\right)}{1-\beta\left(k \pm Q_{ \pm 1}\right)^{2}} \\
q_{ \pm}=-\frac{a\left(k \pm Q_{ \pm 1}\right)}{1-\beta\left(k \pm Q_{ \pm 1}\right)^{2}} \\
v_{ \pm}=\frac{C+2 \beta\left(k \pm Q_{ \pm 1}\right)\left(\omega \pm \Omega_{ \pm 1}\right)}{1-\beta\left(k \pm Q_{ \pm 1}\right)^{2}}, \\
\Omega_{+1,+1}=\Omega_{-1,-1}=\Omega_{+1}, \quad \Omega_{+1,-1}=\Omega_{-1,+1}=\Omega_{-1}, \\
Q_{+1,+1}=Q_{-1,-1}=Q_{+1}, \quad Q_{+1,-1}=Q_{-1,+1}=Q_{-1}
\end{gathered}
$$

Решение уравнения (5) будем искать в виде (см., напримep, [3-5])

$$
\lambda_{ \pm}=\frac{K_{ \pm}}{b T} \operatorname{sech}\left(\frac{t-\frac{z}{V_{0}}}{T}\right) e^{i\left(k_{ \pm} z-\omega_{ \pm} t\right)}
$$

где $K_{ \pm}, k_{ \pm}$и $\omega_{ \pm}-$действительные постоянные, $V_{0}-$ скорость нелинейной волны. Предполагаем, что справедливы неравенства

$$
k_{ \pm} \ll Q_{ \pm 1}, \quad \omega_{ \pm} \ll \Omega_{ \pm 1} .
$$

Подставляя уравнение (9) в уравнения (5), (4) и (2), получаем двухкомпонентный векторный бризер, осциллирующий на СРЧ модифицированного уравнения ВBM (1), в виде

$$
\begin{aligned}
u(z, t) & =\frac{2}{b T} \operatorname{sech}\left(\frac{t-\frac{z}{V_{0}}}{T}\right)\left\{K _ { + } \operatorname { c o s } \left[\left(k+Q_{+1}+k_{+}\right) z\right.\right. \\
& \left.-\left(\omega+\Omega_{+1}+\omega_{+}\right) t\right]+K_{-} \cos \left[\left(k-Q_{-1}+k_{-}\right) z\right. \\
& \left.\left.-\left(\omega-\Omega_{-1}+\omega_{-}\right) t\right]\right\},
\end{aligned}
$$

где $T$ - ширина двухкомпонентного нелинейного импульса,

$$
\begin{gathered}
K_{+}^{2}=\frac{p_{+} q_{-}-2 p_{-} q_{+}}{p_{-} q_{+}-2 p_{+} q_{-}} K_{-}^{2} \\
k_{ \pm}=\frac{V_{0}-v_{ \pm}}{2 p_{ \pm}} \\
\omega_{+}=\frac{p_{+}}{p_{-}} \omega_{-}+\frac{V_{0}^{2}\left(p_{-}^{2}-p_{+}^{2}\right)+v_{-}^{2} p_{+}^{2}-v_{+}^{2} p_{-}^{2}}{4 p_{+} p_{-}^{2}} \\
T^{-2}=V_{0}^{2} \frac{v_{+} k_{+}+k_{+}^{2} p_{+}-\omega_{+}}{p_{+}} \\
b^{2}=\frac{V_{0}^{2} q_{+}}{2 p_{+}}\left(K_{+}^{2}+2 K_{-}^{2}\right)
\end{gathered}
$$

С использованием обобщенной версии ПМР получено двухкомпонентное векторное бризерное решение (11) модифицированного уравнения ВВМ (1). Первый член уравнения (11) - это бризер малой амплитуды, осциллирующий на суммарной частоте $\omega+\Omega_{+1}$ с волновым числом $k+Q_{+1}$ (с учетом уравнений $(10)$ ), а второй член - бризер малой амплитуды, осциллирующий на разностной частоте $\omega-\Omega_{-1}$ с волновым 
числом $k-Q_{-1}$. Нелинейная связь между бризерами характеризуется перекрестными членами $2\left|\lambda_{\mp}\right|^{2} \lambda_{ \pm}$уравнения (5). Параметры нелинейной волны определяются уравнениями (8) и (12). Дисперсионные соотношения и связи между осциллирующими параметрами представлены уравнениями (3), (6) и (7).

Полученный двухкомпонентный векторный бризер (11) идентичен решениям уравнений нелинейной оптики и нелинейной акустики, в частности оптическим и акустическим резонансным векторным 0л-импульсам и нерезонансному двухкомпонентному векторному бризеру в диспергирующей и нелинейной керровской среде [3-5]. Следовательно, полученный результат дает основание сделать вывод, что двухкомпонентный векторный бризер, осциллирующий на СРЧ, характеризует общие нелинейные свойства физических систем, описываемых совершенно разными нелинейными уравнениями, и может формироваться в абсолютно различных физических ситуациях и материалах. Это обстоятельство будет стимулировать не только дальнейшие теоретические и экспериментальные исследования двухкомпонентных векторных бризеров, но и их применение в различных устройствах, в которых используются двухкомпонентные нелинейные уединенные волны.

\section{Конфликт интересов}

Автор заявляет, что у него нет конфликта интересов.

\section{Список литературы}

[1] В.Е. Захаров, С.В. Манаков, С.П. Новиков, Л.П. Питаевский, Теория солитонов (Наука, М., 1980).

[2] T. Taniuti, N. Iajima, J. Math. Phys., 14, 1389 (1973).

[3] Г.Т. Адамашвили, М.Д. Пейкришвили, Р.Р. Коплатадзе, Письма в ЖТФ, 43 (7), 17 (2017).

DOI: 10.21883/PJTF.2017.07.44464.16383

[4] G.T. Adamashvili, Eur. Phys. J. D, 74, 41 (2020). DOI: $10.1140 /$ epjd/e2020-100588-y

[5] G.T. Adamashvili, N.T. Adamashvili, M.D. Peikrishvili, R.R. Koplatadze, Arxiv: 1804.02993v1 (2018).

[6] T.B. Benjamin, J.L. Bona, J.J. Mahony, Phil. Trans. R. Soc. (London) A, 272, 47 (1972).

[7] H. Naher, F.A. Abdullah, A. Bekir, New Trends Math. Sci., 3, 78 (2015).

[8] P.G. Estevez, S. Kuru, J. Negro, L.M. Nieto, Arxiv: 0707.0760v2 (2007)

[9] В.И. Ерофеев, И.С. Павлов, Структурное моделирование метаматериалов (ИПФ РАН, Н. Новгород, 2019).

[10] M.M. Khater, D. Lu, E.H.M. Zahran, Appl. Math. Inf. Sci., 11, 1347 (2017). DOI: 10.18576/amis/110511 